\title{
Probing hot-electron effects in wide area plasmonic surfaces using X-ray photoelectron spectroscopy
}

Sencer Ayas, Andi Cupallari, and Aykutlu Dana

Citation: Appl. Phys. Lett. 105, 221608 (2014);

View online: https://doi.org/10.1063/1.4903295

View Table of Contents: http://aip.scitation.org/toc/apl/105/22

Published by the American Institute of Physics

\section{Articles you may be interested in}

Aluminum-based hot carrier plasmonics

Applied Physics Letters 110, 021117 (2017); 10.1063/1.4973814

Coaxial $\mathrm{Ag} / \mathrm{ZnO} / \mathrm{Ag}$ nanowire for highly sensitive hot-electron photodetection

Applied Physics Letters 106, 081109 (2015); 10.1063/1.4913613

Solar energy conversion via hot electron internal photoemission in metallic nanostructures: Efficiency estimates Journal of Applied Physics 115, 134301 (2014); 10.1063/1.4870040

Surface-plasmon enhanced photodetection at communication band based on hot electrons Journal of Applied Physics 118, 063101 (2015); 10.1063/1.4928133

Probing polarization modes of $\mathrm{Ag}$ nanowires with hot electron detection on $\mathrm{Au} / \mathrm{TiO}_{2}$ nanodiodes Applied Physics Letters 102, 123112 (2013); 10.1063/1.4799156

Long-range Tamm surface plasmons supported by graphene-dielectric metamaterials Journal of Applied Physics 121, 033101 (2017); 10.1063/1.4973900

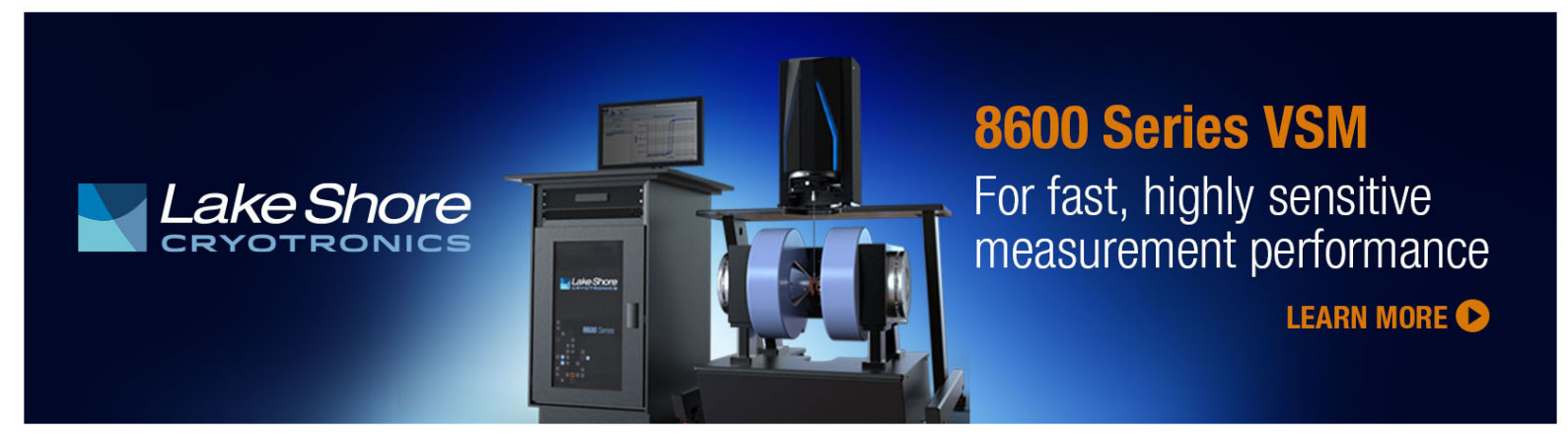




\title{
Probing hot-electron effects in wide area plasmonic surfaces using X-ray photoelectron spectroscopy
}

\author{
Sencer Ayas, ${ }^{\text {a) }}$ Andi Cupallari, ${ }^{\text {a) }}$ and Aykutlu Dana ${ }^{\text {b) }}$ \\ UNAM Institute of Materials Science and Nanotechnology, Bilkent University, 06800 Ankara, Turkey
}

(Received 7 August 2014; accepted 21 November 2014; published online 5 December 2014)

\begin{abstract}
Plasmon enhanced hot carrier formation in metallic nanostructures increasingly attracts attention due to potential applications in photodetection, photocatalysis, and solar energy conversion. Here, hot-electron effects in nanoscale metal-insulator-metal (MIM) structures are investigated using a non-contact X-ray photoelectron spectroscopy based technique using continuous wave X-ray and laser excitations. The effects are observed through shifts of the binding energy of the top metal layer upon excitation with lasers of 445, 532, and $650 \mathrm{~nm}$ wavelength. The shifts are polarization dependent for plasmonic MIM grating structures fabricated by electron beam lithography. Wide area plasmonic MIM surfaces fabricated using a lithography free route by the dewetting of evaporated $\mathrm{Ag}$ on $\mathrm{HfO}_{2}$ exhibit polarization independent optical absorption and surface photovoltage. Using a simple model and making several assumptions about the magnitude of the photoemission current, the responsivity and external quantum efficiency of wide area plasmonic MIM surfaces are estimated as $500 \mathrm{nA} / \mathrm{W}$ and $11 \times 10^{-6}$ for $445 \mathrm{~nm}$ illumination. (C) 2014 AIP Publishing LLC. [http://dx.doi.org/10.1063/1.4903295]
\end{abstract}

Plasmonic structures have attracted increasing attention in the recent years, enabling engineering of optical properties of surfaces. ${ }^{1-3}$ Hot-electron effects (HEE) in plasmonic structures have been investigated as alternative mechanisms for solar energy harvesting and photodetection. ${ }^{4-8}$ HEE rely on transfer of hot carriers from one contact to another through a thin dielectric barrier and provide a semiconductor free route for the photoconversion. A relevant thin film structure is the metal-insulator-metal (MIM) configuration, where optical properties of the surface can be tuned through plasmonic resonances, while separately allowing engineering of carrier transfer and collection. Hot-electron transfer competes with thermalization in the conversion of the absorbed light into a DC or voltage. ${ }^{9,10}$ The transient electron energy distributions of metallic surfaces excited by light pulses are thermalized in short time scales ranging from 100 fs to $1 \mathrm{ps}$. Photoelectron emission with pulsed X-ray, UV, and visible light sources was used in investigation of non-equilibrium energy distributions of carriers on surfaces. ${ }^{11-13}$ Such pulsed or pump-probe measurements provide direct information about carrier dynamics, however require advanced light sources. X-Ray Photoelectron Spectroscopy (XPS) was used to investigate surface photovoltaic and photoconductivity effects in nanocomposite surfaces without using femtosecond or picoseconds light or X-ray pulses. ${ }^{14-18}$ Here, we extend such use of XPS to investigate HEE in plasmonic MIM structures. Laser excitation is used to illuminate the plasmonic surfaces (Figure 1(a)). The top metal of the MIM structures acts as the plasmonic antenna (metal nanodiscs or gratings/stripes) that provides enhanced optical absorption. Plasmonic enhancement leads to more efficient hot-electron generation. HEE result in surface photovoltages and are

\footnotetext{
${ }^{\text {a) }}$ S. Ayas and A. Cupallari contributed equally to this work

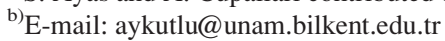

observed by comparing shifts of binding energy (BE) of the top metal islands for dark and illuminated conditions.

A schematic description of the band diagram of a MIM surface under X-ray and visible light illumination is shown in Figure 1(b). A top metal layer, Ag, is separated from a bottom metal layer by a thin dielectric barrier. Under X-ray illumination, the photoelectrons that escape from the top metal islands result in a positive current $\left(\mathrm{J}_{\mathrm{x}}\right)$, charging the surface positively (Figure 1(a)). As a consequence, band bending occurs as shown in Figure 1(b). In the absence of a compensating electron gun, the surface potential reaches steady-state due to the presence of direct (or trap assisted) tunneling current from the bottom metal to the surface, denoted by $J_{t}$ in Figure 1(b). When the surface is illuminated, absorption takes place at the top and bottom metals and the hot-electrons acquire an energy distribution, as shown by rectangles in Figure 1(b) on both sides of the junction. The electron mean free path is on the order of $20-30 \mathrm{~nm}$, which is longer than the dielectric thickness. Therefore, hot-electrons with energies greater than the barrier height can be partially transmitted towards the top metal island where they are thermalized. ${ }^{9}$ This causes a negative shift of the observed BE of Ag. In contrast, hot-electrons generated in the top metal layer are not able to tunnel from the positively charged island.

Recently, a nanostripe antenna was used to demonstrate plasmonic hot-electron current enhancement in MIM devices. ${ }^{23}$ Polarization dependence of the enhancement was measured to show that optical absorption in the metal layers was dominated by plasmonic effects. Here, we demonstrate that HEE related surface photovoltages can be measured in similar MIM structures using XPS. Periodic MIM structures are fabricated using standard techniques. A $70 \mathrm{~nm}$ thick $\mathrm{Ag}$ layer is covered with $5 \mathrm{~nm} \mathrm{HfO}_{2}$, deposited using Atomic Layer Deposition (ALD) at $100^{\circ} \mathrm{C}$. Metal stripes are patterned by electron beam lithography and lift-off, resulting in $50 \mathrm{~nm}$ thick $\mathrm{Ag}$ stripes. The width, period, and length of the stripes 
(a)

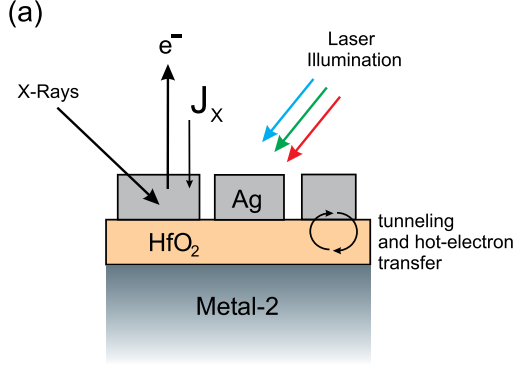

(b)

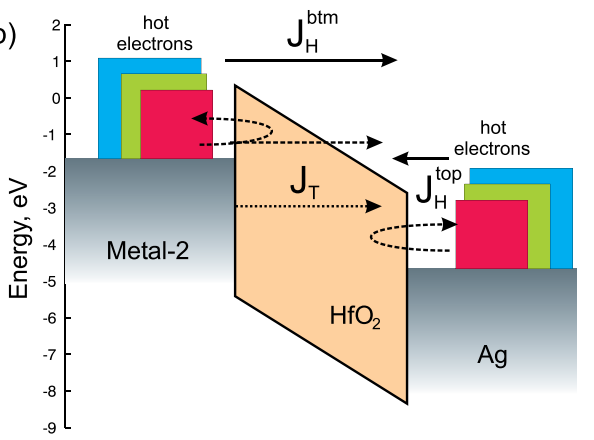

(c)

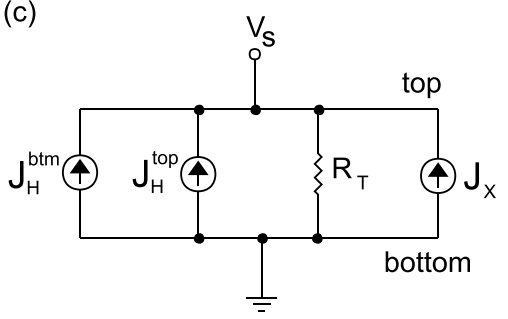

FIG. 1. (a) Cross-section of the MIM surface during an XPS measurement with laser illumination. $\mathrm{J}_{\mathrm{X}}$ represents the X-ray induced photoemission current into the vacuum. (b) The band diagram of the MIM junction under X-ray and laser illumination. Hot-electron energy distributions are shown with rectangles above the Fermi level, due to absorption of light in the top and bottom metal layers. (c) A simplified circuit model with various currents acting on the top metal layer which acquires a steady state voltage of $\mathrm{V}_{\mathrm{s}}$. Current $\mathrm{J}_{\mathrm{T}}$ through the dielectric is modeled within a first order approximation by the resistor $\mathrm{R}_{\mathrm{T}}$.

are $150 \mathrm{~nm}, 250 \mathrm{~nm}$, and $50 \mu \mathrm{m}$, respectively. The area of the patterns $\left(250 \mu \mathrm{m}^{2}\right)$ matches the XPS data collection spot size. In order to elucidate the plasmonic nature of absorption in the metal layers, two grating structures perpendicular to each other are fabricated (Figure 2(a)). The MIM gratings feature wide angle and localized resonances in their optical response. ${ }^{19}$ Field localization is calculated using Finite-Difference TimeDomain (FDTD) simulations for the TE and TM polarizations (Figure 2(b)). The plasmonic absorption $P_{a}$ in the metal layers is resistive and location dependent, $P_{a}(r)=\langle\vec{j}(r) \cdot E(\vec{r})\rangle$ $=\frac{1}{2} \epsilon_{0} \epsilon_{i} \omega|E(r)|^{2}$, where $\vec{j}(r)$ is the current density, $\epsilon_{0}$ is the vacuum electric permittivity, $\epsilon_{i}$ is the imaginary part of the relative electric permittivity of the metal, $\omega$ is the angular frequency, and $\mathrm{E}(\mathrm{r})$ is the local electric field. Total absorption can be calculated by integrating the absorbed power over the material boundaries. It is seen in Figure 2(b) that TE polarization does not excite the plasmonic mode and little or no field penetrates the metal within the gap region. In this case, hot-electron generation is minimal. In contrast, TM polarization excites the plasmonic resonance and enhances hot-electron generation in the top and bottom metal layers, within the regions facing the dielectric gap. The hotelectrons tunnel across the dielectric and disturb the charge equilibrium of the surface Ag layers and cause shifts in the apparent $\mathrm{BE}$ of $\mathrm{Ag}$ under $532 \mathrm{~nm}$ illumination at $40 \mathrm{~mW} / \mathrm{mm}^{2}$ (Figure 2(c)). The workfunction of $\mathrm{Ag}$ is assumed to be $4.7 \mathrm{eV}$. The $\mathrm{HfO}_{2} / \mathrm{Ag}$ conduction band barrier is $2.05 \mathrm{eV}$ and this facilitates partial tunneling of hotelectrons for the used laser wavelengths $445 \mathrm{~nm}(2.77 \mathrm{eV})$, $532 \mathrm{~nm}(2.34 \mathrm{eV})$, and $650 \mathrm{~nm}(1.92 \mathrm{eV}){ }^{20}$ The laser is incident at $45^{\circ}$ and the polarization can be chosen to be parallel or at an angle to the surface plane by a polarizer. Under dark conditions, the $\mathrm{Ag} 3 \mathrm{~d}$ peaks whose native binding energies are $374.35 \mathrm{eV}\left(\mathrm{Ag} 3 \mathrm{~d}_{3 / 2}\right)$ and $368.24 \mathrm{eV}\left(\mathrm{Ag} \mathrm{3d_{5/2 }}\right)$ are shifted towards positive energies (to $370.2 \mathrm{eV}$ for the $\mathrm{Ag}$ $3 \mathrm{~d}_{5 / 2}$ peak) due to X-Ray induced charging. When the polarization has a vertical component, a negative BE shift is observed for the grating that is perpendicular to the light propagation vector (from $370.2 \mathrm{eV}$ to $369.1 \mathrm{eV}$ for the $\mathrm{Ag}$ $3 \mathrm{~d}_{5 / 2}$ peak, II in Figure 2(c)), while the gratings parallel to the excitation (I in Figure 2(c)) do not exhibit a significant shift. When the polarization is rotated, the responses of the periodic stripe antennas are reversed, highlighting the plasmonic origin of the observed shifts.
In order to demonstrate that HEE can be observed in wide area plasmonic surfaces, we use spontaneously organized MIM surfaces (Figure 3(a)). The surfaces are fabricated by depositing $5 \mathrm{~nm}$ thick ALD deposited $\mathrm{HfO}_{2}\left(\right.$ at $100^{\circ} \mathrm{C}$ ) on

(a)

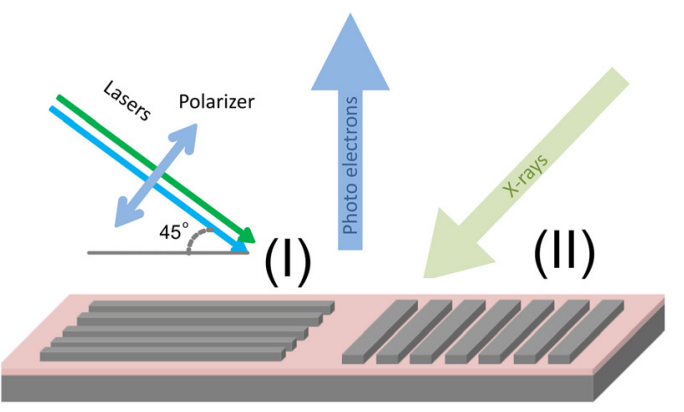

(b)
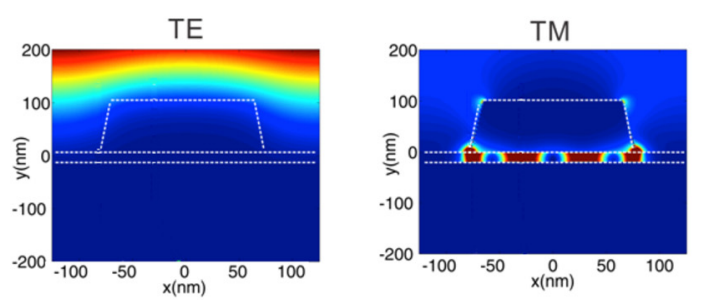

(c)

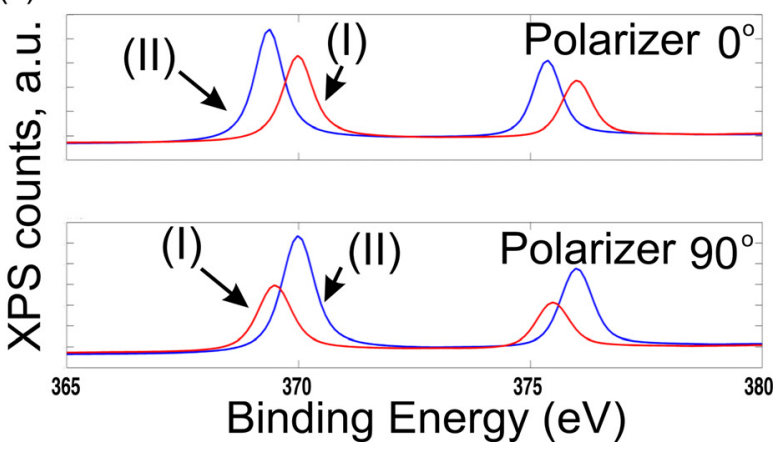

FIG. 2. (a) Schematic description of the MIM grating structures used in polarization dependent measurements. (b) Calculated field profiles of the structures for TE and TM polarizations. Field enhancement is minimal for TE polarization, while plasmonic enhancement is present in the gap for TM polarization. (Scale bar $50 \mathrm{~nm}$ ). (c) XPS spectra of the Ag 3d peaks acquired on gratings labeled as (I) and (II) for two polarizer orientations. As the polarizer is rotated from $0^{\circ}$ to $90^{\circ}$, apparent binding energy shifts of the $\mathrm{Ag} 3 \mathrm{~d}_{3 / 2}$ and $3 \mathrm{~d}_{5 / 2}$ are observed for the gratings with different orientations. Each polarization excites only one of the gratings, for which the plasmonic modes are excited. 
(a)
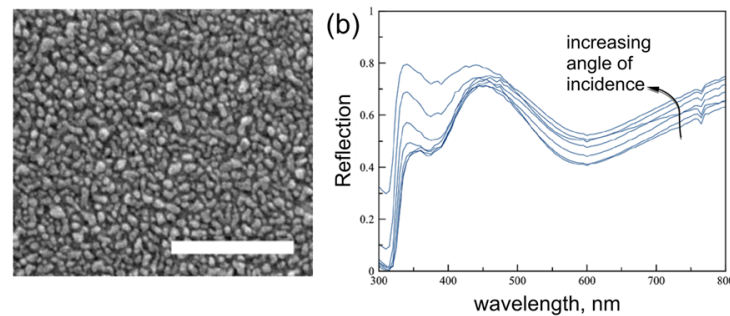

(c)

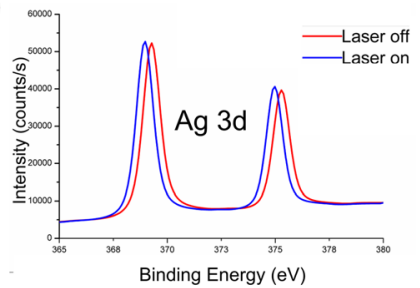

(d)

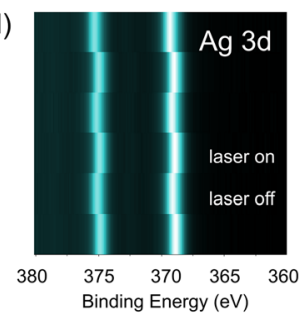

(e)

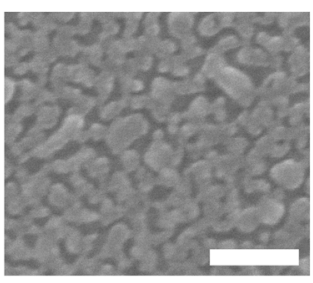

(g)

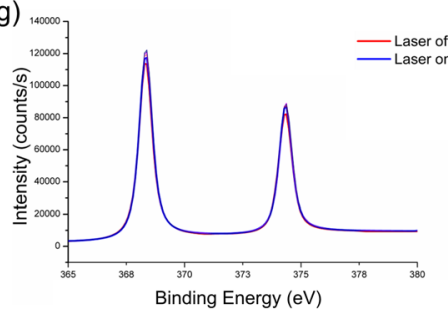

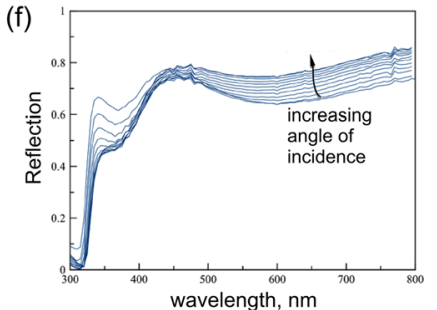

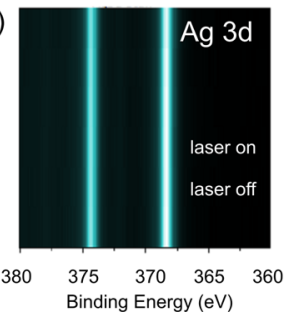

FIG. 3. (a) Scanning electron micrograph of MIM surface fabricated by atomic layer deposition of $\mathrm{HfO}_{2}$ on $\mathrm{Ag}$ and self-organized formation of Ag nanoislands on $\mathrm{HfO}_{2}$ upon $3 \mathrm{~nm}$ thick Ag evaporation (Scale bar $250 \mathrm{~nm}$ ). (b) Reflectance of the surfaces for incidence angles ranging from $20^{\circ}$ to $80^{\circ}$ shows a broad plasmonic absorption peak around $580 \mathrm{~nm}$. (c) Photo-response of the MIM surface when illuminated by $532 \mathrm{~nm}$ excitation, measured by XPS. (d) Consecutive measurements of the XPS spectrum under dark and illuminated conditions exhibit repeatable differential shifts of the Ag 3d lines. (e)-(h) Same as in (a)-(d) except the top Ag mass thickness is $5 \mathrm{~nm}$ and a semicontinuous Ag film is formed instead of MIM nanoislands and no surface photovoltage is observed.

an $80 \mathrm{~nm}$ thick $\mathrm{Ag}$ layer on silicon. A thin Ag layer of $3 \mathrm{~nm}$ mass thickness is evaporated on the $\mathrm{HfO}_{2}$, which forms $\mathrm{Ag}$ nanoislands of average diameter of $30 \mathrm{~nm}$ and thickness of $10 \mathrm{~nm} .^{21,22}$ The surface exhibits a broad plasmonic absorption band around $580 \mathrm{~nm}$ (Figure 3(b)) and a repeatable photoresponse as measured by XPS, as shown in Figures 3(c) and 3(d). When a semicontinuous film is formed on top of $\mathrm{HfO}_{2}$ by depositing $5 \mathrm{~nm}$ thick $\mathrm{Ag}$, no photoresponse can be observed (Figures 3(e)-3(h)). The lack of surface photovoltage in the $5 \mathrm{~nm} \mathrm{Ag}$ case is attributed to reduced plasmonic absorption and lower density of openings in the film where the excitation can couple to into modes between the metal layers. When the surface is illuminated by lasers of wavelength 445,532 , and $650 \mathrm{~nm}\left(22 \mathrm{~mW} / \mathrm{mm}^{2}\right)$, the BE shifts on the $3 \mathrm{~nm}$ Ag surface are observed to be dependent on photon energy (Figure 4(a)). The observed binding energies are shifted from $370.2 \mathrm{eV}$ for the $\mathrm{Ag} 3 \mathrm{~d}_{5 / 2}$ under dark conditions to $369.01 \pm 0.03 \mathrm{eV}$ for $445 \mathrm{~nm}, 369.33 \pm 0.03 \mathrm{eV}$ for $532 \mathrm{~nm}$, and $369.81 \pm 0.03 \mathrm{eV}$ for $650 \mathrm{~nm}$ excitation. The apparent BE is dependent on excitation power (Figure 4(b)) for $445 \mathrm{~nm}$ excitation at 5,10 , and $20 \mathrm{~mW} / \mathrm{mm}^{2}$. The shifts, with respect to observed $\mathrm{BE}$ under dark conditions (at $370.2 \mathrm{eV}$ ), are towards the native (grounded, bulk) BE of $\mathrm{Ag} 3 \mathrm{~d}_{5 / 2}$ at $368.4 \mathrm{eV}$ and increase with increasing excitation power density, saturating at high intensities.

The observations can be understood in terms of steady state currents, including hot-electron, tunneling, and photoemission currents. The IV characteristics of MIM junctions under illumination have been previously studied in the context of energy harvesting and photodetection. If no other internal current sources are present, the open circuit voltage is independent of excitation power and is given by ${ }^{7}$

$$
V_{O C}=-\frac{h \nu-\varphi_{e}}{e} \times\left[1-J_{H}^{b t m} / J_{H}^{t o p}\right]
$$

where $J_{\mathrm{H}}$ 's and $V_{\mathrm{oc}}$ are hot-electron currents and open-circuit voltage, $h \nu$ is the photon energy, $\varphi_{e}$ is the barrier height, and $\mathrm{e}$ is the electronic charge. In the case of the MIM junction illuminated by light and X-rays, the photoelectron current $J_{x}$ must also be included in calculation of the equilibrium condition. For $\mathrm{V}_{\mathrm{S}}>0$, i.e., a net positive charging of the top islands, the hot-electron currents can be written as $J_{H}^{b t m}=-\gamma P_{a}^{b t m}$ $\left(h \nu-\varphi_{e}\right) / h \nu$ and $J_{H}^{t o p}=\gamma P_{a}^{t o p} \max \left(h \nu-\varphi_{e}-e V_{S}, 0\right) / h \nu$, where $P_{a}^{b t m}$ and $P_{a}^{t o p}$ are the absorbed power at the bottom and (a)

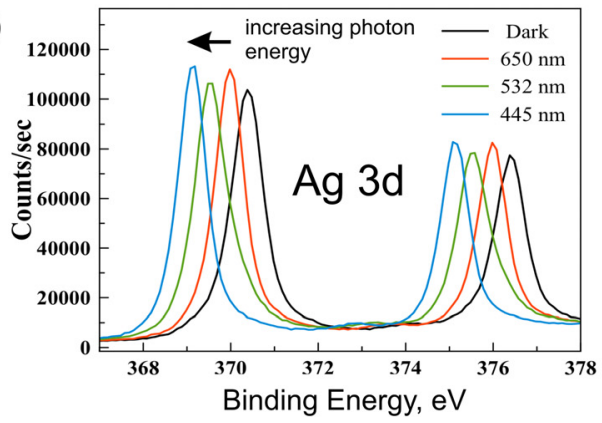

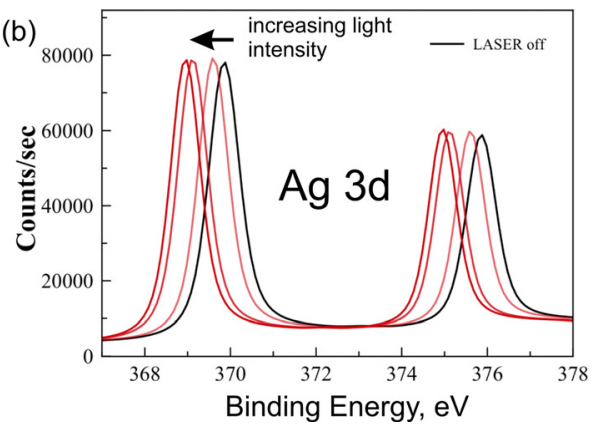

FIG. 4. (a) XPS spectra of the Ag 3d peaks measured on the MIM surface for dark and illuminated conditions, using lasers of 650,532 , and $445 \mathrm{~nm}$ wavelength, $20 \mathrm{~mW}$ power. The binding energies shift to negative due to hot-electron tunneling from the bottom metal to the top Ag island. Greater shifts are observed for increasing photon energy. (b) As the illumination intensity $(445 \mathrm{~nm})$ is increased from 5 to $20 \mathrm{~mW}$, greater negative shift of the binding energy is observed which saturates at high intensities. 
top metal. Here, $\gamma$ is a proportionality constant, which is included to approximately take into account the hot-electron density and velocity inside the metals. Assuming the surface is positively charged, i.e., $\mathrm{V}_{\mathrm{S}}>0$, the circuit diagram in Figure $1(\mathrm{c})$ can be used to infer the steady state surface voltage $\mathrm{V}_{\mathrm{S}}$. For $\mathrm{V}_{\mathrm{S}}$ larger than $\left(E_{p h}-\varphi_{e}\right) / e \approx 0.72 \mathrm{~V}$ for $445 \mathrm{~nm}$ illumination, all hot-electrons from the top metal are assumed to be reflected and $J_{s c}^{t o p} \approx 0$. It was demonstrated that the dielectric behaves as a resistive layer under X-ray exposure, due to continuous ionization within the layer. ${ }^{24,25}$ Resistance of a $4 \mathrm{~nm}$ thick $\mathrm{SiO}_{2}$ layer was determined to be $8.0 \pm 1.0 \mathrm{M} \Omega$ in an XPS measurement specifically designed to extract electronic properties. We assume that the $\mathrm{HfO}_{2}$ layer behaves similarly, like both a tunnel barrier for hot-electrons and a Poole-Frenkel conductor, i.e., $J_{t} \cong V_{S} / R_{t}$. A more accurate quantitative analysis requires direct measurement of the photoelectron current or effective resistance of the $\mathrm{HfO}_{2}$ layer (as done in Ref. 24). The circuit model is still relevant for providing a qualitative understanding of the observed shifts. The steady state condition for $\mathrm{V}_{\mathrm{S}}>0$ is

$$
J_{x}+\gamma P_{a}^{t o p} \frac{\max \left(h \nu-\varphi_{e}-e V_{S}, 0\right)}{h \nu}-\gamma P_{a}^{b t m} \frac{h \nu-\varphi_{e}}{h \nu}=\frac{V_{S}}{R_{T}},
$$

which predicts a surface voltage of $V_{S}=R_{T} J_{x}$ for dark conditions $\left(P_{a}^{t o p}=P_{a}^{b t m}=0\right)$. For the case of high power illumination $\left(P_{a}^{t o p}, P_{a}^{b t m} \rightarrow \infty\right)$, Eq. (2) predicts a surface voltage $V_{S}=\frac{h \nu-\varphi_{e}}{e} \times\left[1-P_{a}^{b t m} / P_{a}^{t o p}\right]$, which is equivalent to the open circuit voltage given in Eq. (1). Using $J_{x} \approx 72 \mathrm{nA} / \mathrm{mm}^{2}$ measured for a similar XPS system, we estimate the resistance of the $5 \mathrm{~nm}$ thick $\mathrm{HfO}_{2}$ layer as $25 \mathrm{M} \Omega$ over $1 \mathrm{~mm}^{2}$ surface area. According to the data shown in Figure 4(b) for $445 \mathrm{~nm}$ excitation, $5 \mathrm{~mW}$ incident power causes a surface voltage shift of $256 \mathrm{mV}$ over a $300 \mu \mathrm{m}$ diameter illumination area, corresponding to a hot-electron current of $2.5 \mathrm{nA}$. The current is used to estimate the external quantum efficiency (EQE) as $11 \times 10^{-6}$ and responsivity as $500 \mathrm{nA} / \mathrm{W}$, for the $300 \mu \mathrm{m}$ diameter measurement area. The calculated responsivity compares favorably with the $75 \mathrm{nA} / \mathrm{W}$ measured for the same wavelength in stripe nanoantenna devices. ${ }^{23} \mathrm{At}$ higher excitation densities at $445 \mathrm{~nm}$ (Figure 4(b)), the surface voltage approaches $0.54 \mathrm{~V}$, which would require a $J_{H}^{b t m} / J_{H}^{t o p}$ ratio of 0.25 according to Eq. (1).

In conclusion, we demonstrated that quasi-static XPS measurements can be used to observe and investigate HEE on plasmonic surfaces. The assumptions about the circuit model element values lead to EQE and responsivity values that are in agreement within the same order of magnitude of previously reported MIM devices. One issue that must be noted is: hot-electron currents are expected to be minimal for red laser excitation $(1.92 \mathrm{eV})$, due to sufficiently large $\mathrm{HfO}_{2}$ conduction band barrier $(2.02 \mathrm{eV})$. The observation of surface photovoltages for this wavelength is either due to reduced conduction band barrier for the $\mathrm{HfO}_{2}$ material deposited in our facility or due to the involvement of hotelectrons that are excited from trap states inside the $\mathrm{HfO}_{2}$ barrier. These observations suggest that the model we present here is basic and more advanced modeling must be used to better understand the dependence of the surface photovoltage on experimental parameters. In a recent article, surface photovoltages have been observed in arrays of gold nanoparticles on an indium tin oxide substrate using Kelvin Probe Force Microscopy (KPFM). ${ }^{26}$ In contrast, the non-contact method we demonstrated here to study surface photovoltages in plasmonic nanostructures does not require a proximal probe and possesses the additional benefit of chemical resolution due to the use of XPS.

This work was partially supported by TUBITAK under Grant No. 111M344, EU FP7: People-IAPP NanoBacterPhageSERS.

${ }^{1}$ S. A. Maier and H. A. Atwater, J. Appl. Phys. 98(1), 011101 (2005).

${ }^{2}$ J. A. Schuller, E. S. Barnard, W. Cai, Y. C. Jun, J. S. White, and M. L. Brongersma, Nat. Mater. 9(3), 193-204 (2010).

${ }^{3}$ H. A. Atwater and A. Polman, Nat. Mater. 9(3), 205-213 (2010).

${ }^{4}$ F. Wang and N. A. Melosh, Nat. Commun. 4, 1711 (2013).

${ }^{5}$ K. Kempa, M. J. Naughton, Z. F. Ren, A. Herczynski, T. Kirkpatrick, J. Rybczynski, and Y. Gao, Appl. Phys. Lett. 95(23), 233121 (2009).

${ }^{6}$ M. A. Green, Prog. Photovoltaics 9(2), 123-135 (2001).

${ }^{7}$ F. Wang and N. A. Melosh, Nano Lett. 11(12), 5426-5430 (2011).

${ }^{8}$ M. W. Knight, Y. Wang, A. S. Urban, A. Sobhani, B. Y. Zheng, P. Nordlander, and N. J. Halas, Nano Lett. 13(4), 1687-1692 (2013).

${ }^{9}$ F. B. Atar, E. Battal, L. E. Aygun, B. Daglar, M. Bayindir, and A. K. Okyay, Opt. Express 21(6), 7196-7201 (2013).

${ }^{10}$ T. P. White and K. R. Catchpole, Appl. Phys. Lett. 101(7), 073905 (2012).

${ }^{11}$ Y. H. Wang, H. Steinberg, P. Jarillo-Herrero, and N. Gedik, Science 342(6157), 453-457 (2013).

${ }^{12}$ J. Cao, Y. Gao, R. J. D. Miller, H. E. Elsayed-Ali, and D. A. Mantell, Phys. Rev. B 56(3), 1099 (1997).

${ }^{13}$ M. Bauer, C. Lei, K. Read, R. Tobey, J. Gland, M. M. Murnane, and H. C. Kapteyn, Phys. Rev. Lett. 87(2), 025501 (2001).

${ }^{14}$ O. O. Ekiz, K. Mizrak, and A. Dana, ACS Nano 4(4), 1851-1860 (2010).

${ }^{15}$ H. Sezen, A. A. Rockett, and S. Suzer, Anal. Chem. 84(6), 2990-2994 (2012).

${ }^{16}$ H. Sezen and S. Suzer, J. Vac. Sci. Technol., A 28(4), 639-642 (2010).

${ }^{17}$ S. Suzer and A. Dana, J. Phys. Chem. B 110(39), 19112-19115 (2006).

${ }^{18}$ S. Suzer, H. Sezen, and A. Dana, Anal. Chem. 80(10), 3931-3936 (2008).

${ }^{19}$ S. Ayas, H. Guner, B. Türker, O. O. Ekiz, F. Dirisaglik, A. K. Okyay, and A. Dana, ACS Nano 6(8), 6852-6861 (2012).

${ }^{20}$ M. L. Huang, Y. C. Chang, Y. H. Chang, T. D. Lin, J. Kwo, and M. Hong, Appl. Phys. Lett. 94(5), 052106 (2009).

${ }^{21}$ S. Ayas, A. Cupallari, O. O. Ekiz, Y. Kaya, and A. Dana, ACS Photonics 1(1), 17-26 (2013).

${ }^{22}$ S. Ayas, G. Cinar, A. D. Ozkan, Z. Soran, O. O. Ekiz, D. Kocaay, and A. Dana, Sci. Rep. 3, 2624 (2013).

${ }^{23}$ H. Chalabi, D. Schoen, and M. L. Brongersma, Nano Lett. 14(3), 1374-1380 (2014).

${ }^{24}$ G. Ertas, U. K. Demirok, A. Atalar, and S. Suzer, Appl. Phys. Lett. 86(18), 183110 (2005).

${ }^{25}$ H. Cohen, Appl. Phys. Lett. 85(7), 1271-1273 (2004).

${ }^{26}$ M. T. Sheldon, J. van de Groep, A. M. Brown, A. Polman, and H. A. Atwater, Science 346, 828-831 (2014). 